\title{
Ergosterol limits osteoarthritis development and progression through activation of $\mathrm{Nrf} 2$ signaling
}

\author{
DAWEI CAI ${ }^{1}$, HUYONG YAN ${ }^{2}$, JUN LIU $^{3}$, SICHUN CHEN $^{1}$, LONGHAI JIANG $^{1}$, XIAOXU WANG ${ }^{2}$ and JIAN QIN ${ }^{1}$ \\ ${ }^{1}$ Department of Orthopaedics, Sir Run Run Hospital, Nanjing Medical University, Nanjing, \\ Jiangsu 211100; ${ }^{2}$ Department of Orthopaedics, The Second Affiliated Hospital of Nanhua \\ University, Hengyang, Hunan 421000; ${ }^{3}$ Department of Orthopaedics, The Second Affiliated \\ Hospital of Nanjing Medical University, Nanjing, Jiangsu 210011, P.R. China
}

Received May 13, 2020; Accepted November 4, 2020

DOI: $10.3892 /$ etm.2021.9627

\begin{abstract}
Osteoarthritis (OA) is a common joint disorder characterized by progressive articular cartilage degeneration and destruction and results in gradual disability among middle-aged and elderly patients. Our previous study demonstrated that depletion of nuclear factor erythroid 2-related factor 2 (Nrf2) exacerbated cartilage erosion in an $\mathrm{OA}$ model and that activation of the Nrf2 pathway could counter this process. As a downstream target of Nrf2, heme oxygenase (HO) degrades heme to free iron, biliverdin and carbon monoxide $(\mathrm{CO})$, which protects against oxidative stress. Ergosterol (ER), which is extracted from fungi, is a newly discovered Nrf2 activator and displayed efficacy against myocardial injury. The present study aimed to investigate the potential protective effects of ER against cartilage damage during OA. Primary mouse chondrocytes were treated with ER for in vitro assays. Furthermore, mice that underwent destabilization of the medial meniscus surgery were orally administered with ER. Western blotting suggested that ER increased protein expression of Nrf2 and HO-1 in primary chondrocytes and articular cartilage from knee joints. Cartilage damage in knee joints was significantly reduced by ER treatment. Western blotting and PCR analysis confirmed that ER could also suppress the expression of MMP-9 and MMP-13 in vivo and in vitro. The present findings suggested that ER effectively alleviated cartilage degradation and that activation of the Nrf2-heme
\end{abstract}

Correspondence to: Professor Xiaoxu Wang, Department of Orthopaedics, The Second Affiliated Hospital of Nanhua University, 35 Jiefang Road, Hengyang, Hunan 421000, P.R. China

E-mail: wxx1024@163.com

Professor Jian Qin, Department of Orthopaedics, Sir Run Run Hospital, Nanjing Medical University, 109 Longmian Road, Nanjing, Jiangsu 211100, P.R. China

E-mail: qinjian@njmu.edu.cn

Key words: ergosterol, osteoarthritis, nuclear factor erythroid 2-related factor 2, chondrocytes oxygenase 1 pathway may play a role in ER-mediated cartilage protection against $\mathrm{OA}$.

\section{Introduction}

Osteoarthritis (OA) is an aging-associated progressive joint disease characterized by cartilage loss and damage $(1,2)$. However, to date, there is no effective and safe medication available against OA. Most patients with advanced OA require total joint replacement (3). Recently, it was hypothesized that oxidative stress is closely associated with the progression of cartilage degeneration during OA, and promotion of endogenous antioxidant activity was demonstrated to confer protection against OA (4-8). Nuclear factor erythroid 2-related factor 2 (Nrf2) is a transcription factor, which binds to antioxidant response elements (AREs) to regulate numerous phase II antioxidant enzymes, including heme oxygenase 1 (HO-1) (9). Our previous study showed that Nrf2 deficiency aggravated the damage of cartilage in an inflammatory model and a post-traumatic model of OA (10). Therefore, the present study aimed to investigate potential drugs targeting Nrf2 activation. Ergosterol (ER), which is extracted from the fungus Agaricus campestris, exhibits a wide range of pharmacological properties, including anti-inflammatory and anti-oxidative effects $(11,12)$. Xu et al (13) reported that ER increased the expression of Nrf2 and HO-1 in rat hearts and exerted a cardioprotective effect in a lipopolysaccharide-induced sepsis model. However, to the best of our knowledge, the role of ER in OA remains unclear. The aim of the present study was to investigate the chondroprotective effects of ER in a destabilization of the medial meniscus (DMM) surgery-induced OA model and elucidate the underlying mechanisms.

\section{Materials and methods}

Chemicals and reagents. ER (cat. no. 45480; purity, 95\%) was supplied by Sigma-Aldrich; Merck KGaA. Anti-Nrf2 (cat. no. 12721; 1:1,000), anti-lamin B (cat. no. 13435; 1:1,000) and anti-GAPDH (cat. no. 5174; 1:5,000) antibodies were purchased from Cell Signaling Technology, Inc.. Anti-HO-1 (cat. no. BS6626; 1:1,000), anti-MMP1 (cat. no. BS62563; 1:1,000), anti-MMP3 (cat. no. BS90872; 1:1,000), anti-MMP9 
(cat. no. BS6893; 1:1,000), anti-MMP13 (cat. no. BS6668; 1:1,000), anti-ADAMTS5 (cat. no. BS74041; 1:2,000) and secondary HRP-conjugated goat anti-rabbit (cat. no. BS13278; 1:5,000) antibodies were purchased from Bioworld Technology, Inc.. A nuclear and cytoplasmic protein extraction kit (cat. no. P0028) was obtained from Beyotime Institute of Biotechnology.

Cell culture. Primary mouse chondrocytes were collected from the costal cartilage of 14 male neonatal C57BL/6 mice (age, 6 days; weight, $3.08 \pm 0.85 \mathrm{~g}$ ) obtained from the Comparative Medical Center of Yangzhou University (Yangzhou, China) and housed in specific-pathogen-free conditions under a $12 \mathrm{~h}$ light-dark cycle at $25 \pm 2^{\circ} \mathrm{C}$ with $45-50 \%$ humidity with food and water available ad libitum. Murine cartilage was collected and collagen II immunofluorescence staining was used for identification of chondrocytes, as previously described (14). Following digestion with collagenase D overnight at $37^{\circ} \mathrm{C}$, harvested chondrocytes were seeded on a $10-\mathrm{cm}$ dish and incubated in DMEM/F12 (Gibco; Thermo Fisher Scientific, Inc.) with $10 \%$ FBS (Gibco; Thermo Fisher Scientific, Inc.), $100 \mathrm{U} / \mathrm{ml}$ penicillin and $100 \mu \mathrm{g} / \mathrm{ml}$ streptomycin with $5 \% \mathrm{CO}_{2}$ at $37^{\circ} \mathrm{C}$. To avoid loss of the chondrocyte phenotype with successive passages, cells at $80 \%$ confluence were detached and plated in six-well plates at a density of $3 \times 10^{5}$ cells/well $(2.4 \mathrm{~cm}$ in diameter) for further assays. Primary mouse chondrocytes pre-treated with IL-1 $\beta(10 \mathrm{ng} / \mathrm{ml}$; as previously described) $(15,16)$ for $12 \mathrm{~h}$ were cultured in various concentrations $(0,5,10$ or $20 \mu \mathrm{M})$ of ER for $24 \mathrm{~h}$ at $37^{\circ} \mathrm{C}$ followed by protein or RNA extraction.

Cell viability assays. A Cell Counting Kit-8 (CCK-8) assay was used to determine cell viability following ER treatment, as detailed in our previous study (14). Briefly, primary chondrocytes were seeded in a 96-well plate at a density of $5 \times 10^{3}$ cells/well. Following incubation with different concentrations $(0,2,5,10,20$ or $40 \mu \mathrm{M})$ of ER at $37^{\circ} \mathrm{C}$ for $24 \mathrm{~h}$, cells were incubated with CCK-8 solution (Bioworld Technology, Inc; $10 \mu \mathrm{l} /$ well) for $1 \mathrm{~h}$ in the dark at room temperature. The absorbance of each well was subsequently measured using a microplate reader (Bio-Rad Laboratories, Inc.) at a wavelength of $450 \mathrm{~nm}$ according to the manufacturer's protocols.

Protein expression analysis. Male mice were kept at $24^{\circ} \mathrm{C}$ with a 12-h light/dark cycle and food and water access ad libitum. Mice were monitored for health and weight every 2 or 3 days. In total 68 knees from 34 mice were used for protein expression analysis. OA was induced by sectioning of the medial meniscotibial ligament, also known DMM surgery. The pre-surgery grouping was separate from the surgery grouping. For pre-surgery, two groups of mice ( $n=8$ per group) that had undergone no sham or DMM surgery were administered saline (vehicle) or ER $(25 \mathrm{mg} / \mathrm{kg} /$ day) dissolved in saline $(0.1 \mathrm{ml} / 10 \mathrm{~g})$ every day by oral gavage for 2 weeks, and 16 knee joints were harvested for WB analysis to confirm whether ER could increase expression of Nrf2/HO-1 in cartilage. For surgery groups, two groups of mice ( $n=9$ per group) that had undergone DMM surgery on the right knee and sham surgery on the left knee were administered the aforementioned doses of vehicle or ER for 2 weeks. At the eighth week after surgery, atotal of 36 knees were harvested and formed the four groups (sham surgery + vehicle, sham surgery + ER, DMM surgery + vehicle and DMM surgery + ER). The knees were prepared as described previously (17). Briefly, cartilage tissue obtained from knee joints was harvested using a scalpel blade with a surgical microscope and stored in liquid nitrogen. To obtain a suitable amount of protein, sample pooling was performed. The cartilage collected from each individual knee (including a femur and tibia) was treated as one compartment. Each experimental unit of mouse samples was pooled from three compartments from different mice. When pooling was performed, the experimental unit was regarded as one sample. For animal tissues, the cartilage tissue was extracted in PBS containing 1\% Triton X-100, 0.1\% SDS, $20 \mathrm{nM}$ sodium orthovanadate, $1 \mu \mathrm{g} / \mathrm{ml}$ aprotinin, $1 \mathrm{mM}$ phenylmethylsulfonyl fluoride and $5 \mathrm{mM}$ ethylenediaminetetraacetic acid. The homogenates were centrifuged at $12,000 \mathrm{x}$ g for $30 \mathrm{~min}$ at $4^{\circ} \mathrm{C}$ and the protein in the supernatant was used for further study. When analyzing fractionated protein from cultured cells the cytoplasmic and nuclear fractions were separated using the aforementioned nuclear and cytoplasmic protein extraction kit according to the manufacturer's instruction. Briefly, the cultured chondrocytes were washed twice with pre-cooled PBS and harvested by centrifugation at $1,000 \mathrm{x} g$ for $5 \mathrm{~min}$ at $4^{\circ} \mathrm{C}$, and the cell pellet was lysed in a cytoplasmic extraction reagent. After incubation for $30 \mathrm{~min}$ on ice, the homogenate was centrifuged at $10,000 \mathrm{x}$ g for $10 \mathrm{~min}$ at $4^{\circ} \mathrm{C}$ and the supernatant was removed. The nuclear extraction reagent was subsequently added to the precipitate. After incubation for $30 \mathrm{~min}$ on ice, nuclear protein fraction was harvested in the supernatant after centrifugation at $10,000 \mathrm{x}$ g for $10 \mathrm{~min}$ at $4^{\circ} \mathrm{C}$. In a separate total protein extraction, cultured chondrocytes were lysed with pre-cooled RIPA lysis buffer (Sigma-Aldrich; Merck KGaA cat. no. R0278) containing protease and phosphatase inhibitors. The protein concentration of the lysates was measured with a bicinchoninic acid protein quantitation kit (Pierce; Thermo Fisher Scientific, Inc.). Equal amounts of protein $(5 \mu \mathrm{g}$ of protein per lane for tissues and $10 \mu \mathrm{g}$ of protein per lane for cells) were loaded on SDS-PAGE (10\% gel) and transferred onto polyvinylidene fluoride membranes (EMD Millipore). The membranes were blocked with 5\% BSA (Sigma-Aldrich; Merck $\mathrm{KGaA}$ ) at room temperature for $1 \mathrm{~h}$ and then incubated with the aforementioned primary antibodies for $18 \mathrm{~h}$ at $4^{\circ} \mathrm{C}$. Following three washes and probing with the aforementioned secondary HRP-conjugated goat anti-rabbit antibodies for $2 \mathrm{~h}$ at $4^{\circ} \mathrm{C}$, membranes were visualized using an Pierce ${ }^{\mathrm{TM}} \mathrm{ECL}$ Western Blotting Substrate (Thermo Fisher Scientific, Inc. cat. no. 32209) and quantified using ImageJ software (version 1.51; National Institutes of Health).

Gene transcript analysis. In total 24 knees were harvested from 12 mice for gene transcript analysis. Pooling was performed to obtain a suitable amount of cartilage and each experimental unit was a pool of two compartments. The cartilage collected from each individual knee (including a femur and tibia) was treated as one compartment. Total RNA from cartilage in knee joints of mice was isolated with TRIzol ${ }^{\circledR}$ reagent (Invitrogen; Thermo Fisher Scientific, Inc.). First strand cDNA was synthesized from total RNA using the PrimeScript RT Reagent kit (Promega Corporation) according 
Table I. Gene-specific primer sequences used for the quantitative PCR.

Target gene

MMP-9

MMP-13

ACTB
Primer sequence $\left(5^{\prime}-3^{\prime}\right)$

Forward: TGGCTTTTGTGACAGGCACTTC

Reverse: CGGTGGTGTTCTCCAATGTAAGAG

Forward: ATGCATTCAGCTATCCTGGCCA

Reverse: AAGATTGCATTTCTCGGAGCCTG

Forward: TGACGGGGTCACCCACACTGTGCCCATCTA

Reverse: CTAGAAGCATTTGCGGTGGACGATGGAGGG to the manufacturer's protocols. mRNA expression of MMP-9 and MMP-13 was measured on a 7500 Real-Rime PCR system with SYBR Green PCR Master Mix (Thermo Fisher Scientific, Inc.). ACTB was used as a reference gene (18). Gene-specific primer sequences used in the present study are listed in Table I. The expression levels of genes were calculated using the $2^{-\Delta \Delta \mathrm{Cq}}$ method (19).

Luciferase assays. The HO-1 promoter was amplified by PCR from RAW cell genome DNA and the product was then inserted into a pGL3 vector (Promega Corporation) at the HindIII and BglII sites (20). DNA from RAW cells was extracted using the TIANamp Genomic DNA Kit (cat. no. DP304; Tiangen Biotech Co., Ltd.) and amplified with AmpliTaq Gold ${ }^{\mathrm{TM}}$ DNA Polymerase (Thermo Fisher Scientific, Inc.) following the manufacturer's instructions. The thermocycling conditions were as follows: $2 \mathrm{~min}$ at $94^{\circ} \mathrm{C} ; 30$ cycles of $30 \mathrm{sec}$ at $94^{\circ} \mathrm{C}$, $30 \mathrm{sec}$ at $56^{\circ} \mathrm{C}$ and $30 \mathrm{sec}$ at $72^{\circ} \mathrm{C}$, and a final $5 \mathrm{~min}$ at $72^{\circ} \mathrm{C}$. All the constructs were subjected to $4 \%$ agarose gel electrophoresis in an ABI Prism 377 DNA sequencer (Applied Biosystems; Thermo Fisher Scientific, Inc.). A nonspecific oligonucleotide was used to construct a control plasmid. The primer sequences for HO-1 were as follows: Forward, 5'-GGAAGA TCTCTGCAGAGCCCCACTGGA G-3' and reverse, 5'-CCC AAGCTTGGAACAGCAACGCTGT-3'. All the constructs were confirmed by sequencing. 293 cells (The Cell Bank of Type Culture Collection of the Chinese Academy of Sciences) were transfected with the HO-1-ARE-promoter-driven luciferase plasmids using Lipofectamine ${ }^{\circledR} 2000$ (Invitrogen; Thermo Fisher Scientific, Inc.) according to the manufacturer's instructions. Following transfection for $24 \mathrm{~h}$, the cells $\left(5 \times 10^{3}\right.$ cells/well in a 96 well plate) were treated with different concentrations of ER $(0,10$ or $20 \mu \mathrm{M})$ for $24 \mathrm{~h}$ at $37^{\circ} \mathrm{C}$. The Dual-luciferase reporter assay system (Promega Corporation) was used to measure luciferase activity by normalizing firefly luciferase activity to Renilla luciferase activity.

OA model and histological analysis. A total of 86 C57BL/6 male mice (age, 10 weeks; weight, $24.78 \pm 4.05 \mathrm{~g}$ ) purchased from the Comparative Medical Center of Yangzhou University (Yangzhou, China) were kept at $24^{\circ} \mathrm{C}$ in standard mouse cages (5 animals per cage) with a 12-h light/dark cycle and food and water access ad libitum. Mice were monitored for health and weight every 2 or 3 days. When pain or distress were observed, the animals were treated with buprenex $(0.1-2.0 \mathrm{mg} / \mathrm{kg}$; Reckitt \& Colman Pharmaceuticals, Inc.), which was added to crushed or wet food. If pain or distress continued, the mice were sacrificed regardless of the scheduled endpoints. The criteria that determined discomfort/distress/pain were any three of the following signs: Abnormal posture, slow, careful or abnormal (waddling) gait, low activity levels, slow eating, cowering or vocalizing on handling, change in eye or coat appearance and weight loss. Animal death following sacrifice was confirmed by one of the following criteria: No response to tail or toe pinch, no respiration or heartbeat following continuous monitoring for $30 \mathrm{sec}$ or rigor mortis. Mice were euthanized using $100 \% \mathrm{CO}_{2}$ anesthesia using an air displacement rate of $20 \%$ of the chamber volume/min. Experiment duration was 8 weeks. The animal research was performed in accordance with Nanjing Medical University Institutional Animal Care and Use Committee guidelines (approval no. IACUC 1903044). The mice were anesthetized with intraperitoneal ketamine hydrochloride $(120 \mathrm{mg} / \mathrm{kg})$ and xylazine hydrochloride $(5 \mathrm{mg} / \mathrm{kg})$. OA was induced by sectioning of the medial meniscotibial ligament, also known as the coronary ligament, which anchors the medial meniscus (MM) to the tibial plateau (21). Forty mice were divided into four groups with 10 mice per group (sham surgery + vehicle, sham surgery + ER, DMM surgery + vehicle and DMM surgery + ER). The sham surgery (ligament was exposed but not transected) was performed on the left knee of the same mice that underwent DMM on the right knee. Mice were administered with saline as vehicle or ER $(25 \mathrm{mg} / \mathrm{kg} /$ day) dissolved in saline by oral gavage for 2 weeks immediately after surgery. Mice were sacrificed at 8 weeks post-DMM surgery. Knee joints were dissected free of skin or excess muscle and fixed with $10 \%$ buffered formalin for $24 \mathrm{~h}$ at $22^{\circ} \mathrm{C}$. Obtained sections $(5 \mu \mathrm{m})$ were placed in $70 \%$ ethyl alcohol for $15 \mathrm{~min}$ and then stained with $0.04 \%$ safranin $\mathrm{O} /$ sodium acetate buffer $(\mathrm{pH} 4.0)$ for $10 \mathrm{~min}$ at $22^{\circ} \mathrm{C}$. Sectioned murine joint tissues were observed under an Olympus BX51 light microscope and photographed by a computer-operated Olympus DP72 digital camera (Olympus Corporation). Sections of knee joints (10 slides per joint) were evaluated by an assessor experienced in this technique and blinded to the origin of the sample using the Osteoarthritis Research Society International scoring system (0-6 subjective scoring system) where the higher the score, the more severe the joint degeneration (22).

Statistical analysis. All data are presented as the mean \pm SEM. All assays were repeated at least three times independently. Statistical analysis was performed using Mann-Whitney 
A<smiles>CC(C)[C@H](C)/C=C/[C@H](C)[C@H]1CC[C@H]2C3=CC=C4C[C@@H](O)CC[C@]4(C)[C@H]3CC[C@]21C</smiles>

D
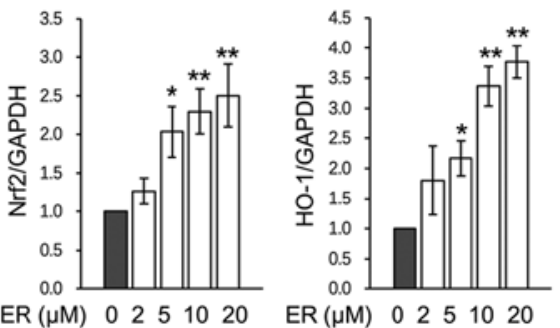

G

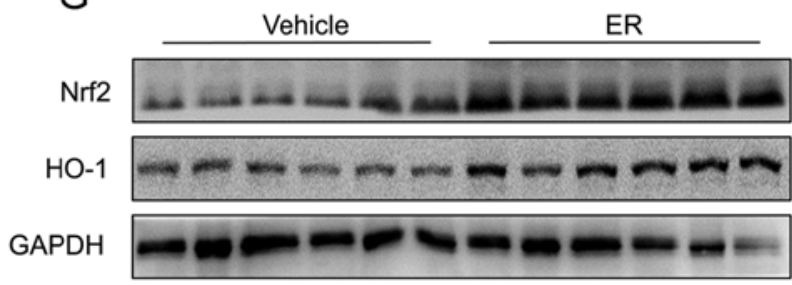

B

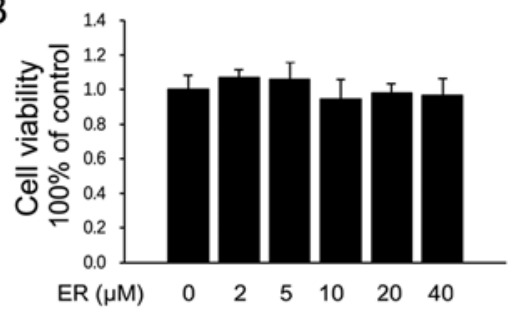

E
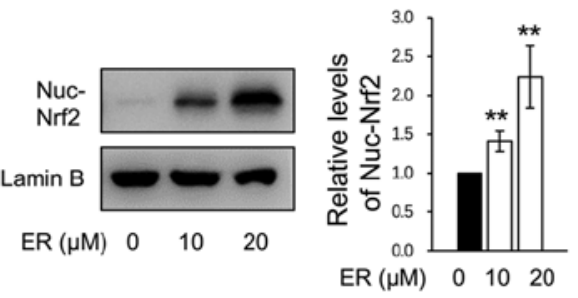

C

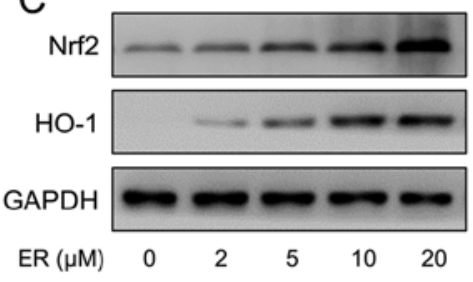

$\mathrm{F}$

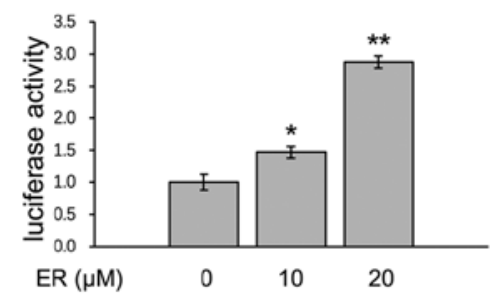

$\mathrm{H}$

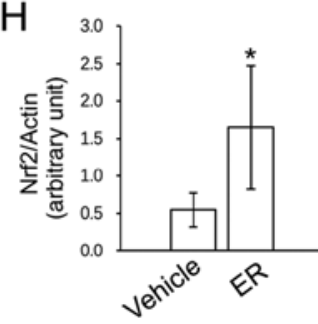

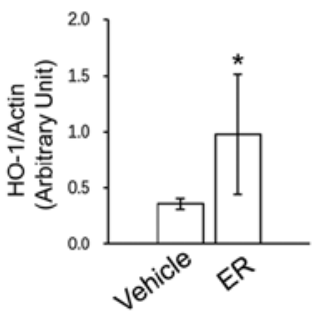

Figure 1. ER activates the Nrf2 signaling pathway in vitro and in vivo. (A) Structure of ER. (B) Cell viability assay. (C) Nrf2 and HO-1 expression levels were assessed using western blot analysis. (D) Quantitative analysis of Nrf2 and HO-1 protein levels. ${ }^{*} \mathrm{P}<0.05$ and ${ }^{* *} \mathrm{P}<0.01$ vs. untreated control. (E) Cell nuclear fractions were isolated from primary murine chondrocytes for western blot analysis. ${ }^{* *} \mathrm{P}<0.01$ vs. untreated control. (F) Luciferase assay using 293 cells transfected with a Renilla luciferase plasmid and an HO-1 promoter reporter plasmid, followed by treatment with increasing doses of ER for $24 \mathrm{~h}$. ${ }^{*} \mathrm{P}<0.05$ and ${ }^{*} \mathrm{P}<0.01$ vs. plasmid control. (G) Western blot analysis of Nrf2 and HO-1 expression from the cartilage of knee joints from vehicle- and ER-treated groups (H) Quantitative analysis of Nrf2 and HO-1 protein levels. ${ }^{* *} \mathrm{P}<0.05$ vs. vehicle-treated group. ER, ergosterol; Nrf2, nuclear factor erythroid 2-related factor 2; HO-1, heme oxygenase 1; vehicle, saline.

U test or one-way ANOVA followed by Tukey's test using GraphPad Prism software (version 6.01; GraphPad Software, Inc.). Datasets containing a mixture of paired and unpaired samples were analyzed using mixed ANOVA followed by Bonferroni correction using SPSS 22.0 (SPSS, IBM Inc.). $\mathrm{P}<0.05$ was considered to indicate a statistically significant difference.

\section{Results}

ER activates the Nrf2 pathway in chondrocytes and cartilage. The chemical structure of ER is presented in Fig. 1A. The cytotoxicity of ER was measured by CCK-8 assay, and concentrations $<40 \mu \mathrm{M}$ were used in primary murine chondrocytes (Fig. 1B). ER significantly increased the protein expression of Nrf2 and HO-1 in chondrocytes in a dose-dependent manner (Fig. 1C and D). Nuclear protein was extracted for assays and the results showed that ER upregulated nuclear Nrf2 expression (Fig. 1E). ER treatment caused a significant increase in luciferase activity (Fig. 1F), which indicated that ER activated the HO-1 promoter transactivation activity. To investigate the effects of ER on Nrf2 and HO-1 expression in the cartilage of knee joints, mice that had undergone no treatment were administered ER ( $25 \mathrm{mg} / \mathrm{kg} /$ day) for 2 weeks and cartilage samples were harvested for western blot analysis. The results revealed that the expression levels of both of Nrf2 and HO-1 were significantly higher in the cartilage of the ER-treated group compared with the expression levels in the saline-treated group (Fig. $1 \mathrm{G}$ and $\mathrm{H}$ ).

ER inhibits the expression of MMPs in chondrocytes. MMPs and a disintegrin and metalloproteinase with thrombospondin motifs (ADAMTS) play a role in cartilage destruction during OA. Both MMP-9 and MMP-13 are important proteolytic enzymes in ECM breakdown, and abnormal up-regulation of these enzymes can induce excess catabolism in cartilage, gradually leading to cartilage breaking down $(23,24)$. To investigate the potential therapeutic effects of ER on OA, the protein expression of matrix-degrading enzymes were further examined. As predicted, IL-1 $\beta$ increased the protein expression levels of MMP-1, MMP-3, MMP-9, MMP-13 and ADAMTS-5 in the cells (Fig. 2). Although IL-1 $\beta$ induced expression of MMP-1 and MMP-3 were not regulated by ER (Fig. 2A-C), significant inhibition of matrix-degrading enzymes MMP-9 and MMP-13 was observed at the protein level (Fig. 2D and E). ADAMTS-5 expression also did not appear to be regulated by ER (Fig. 2F). Considering their roles in the cartilage degradation network $(25,26)$, it was hypothesized that ER may suppress MMP-9 and MMP-13 expression in chondrocytes and reduce cartilage breakdown.

ER alleviates cartilage erosion in experimental $O A$. To determine the protective effects of ER against OA, a murine model of OA was induced by transecting the medial meniscotibial 

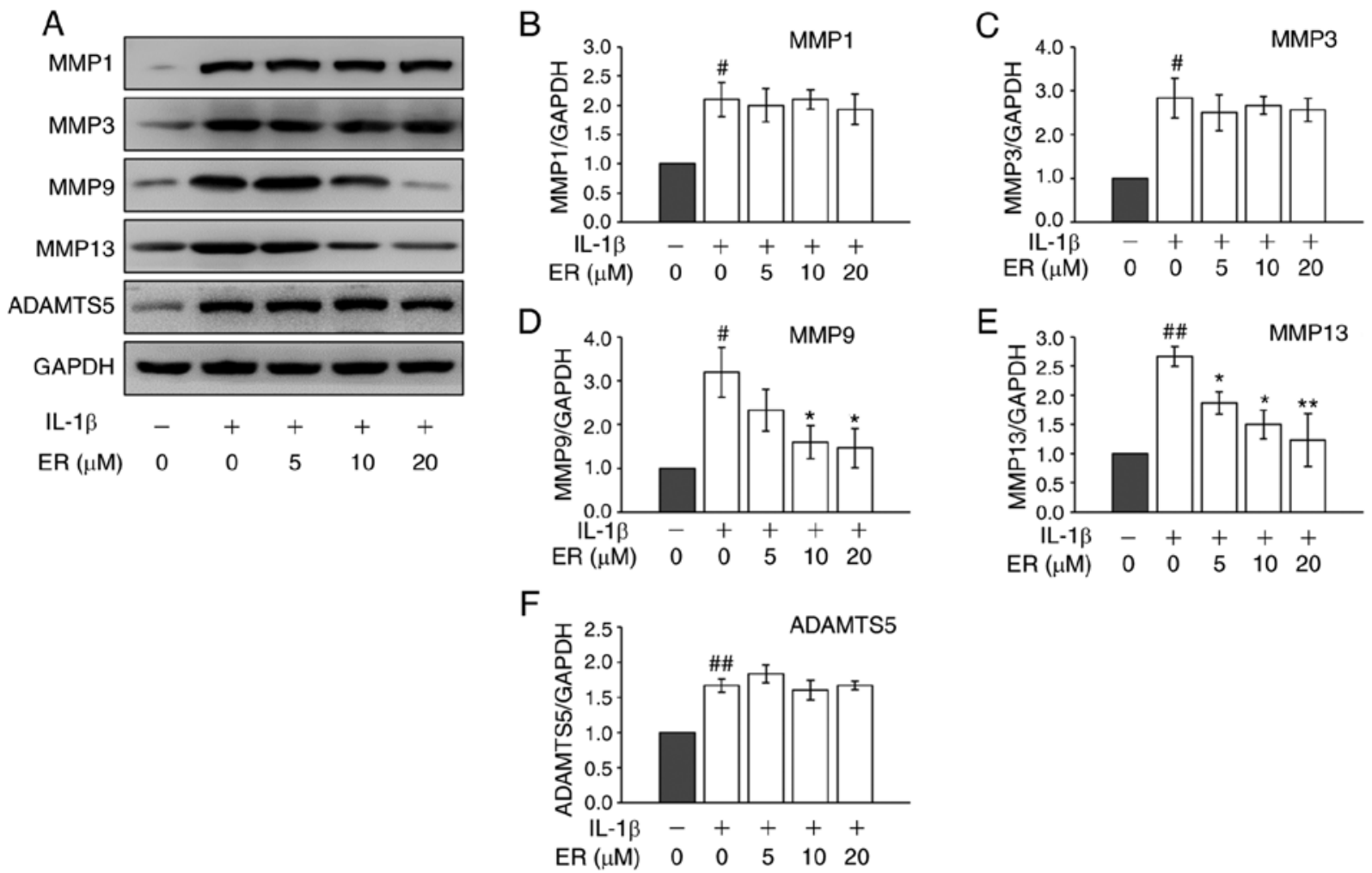

Figure 2. Effects of ER on IL-1 $\beta$-induced MMPs and ADAMTS-5 expression. (A) Protein expression levels of MMPs and ADAMTSs were determined by western blot analysis. Quantitative analysis of (B) MMP1, (C) MMP3, (D) MMP9, (E) MMP13 and (F) ADAMTS5 levels. Bands are representative of three separate experiments. ${ }^{\#} \mathrm{P}<0.05$ and ${ }^{\# \#} \mathrm{P}<0.01$ vs. untreated control. ${ }^{*} \mathrm{P}<0.05$ and ${ }^{* *} \mathrm{P}<0.01$ vs. IL-1 $\beta$ treatment alone. ER, ergosterol; ADAMTS, and a disintegrin and metalloproteinase with thrombospondin motifs.

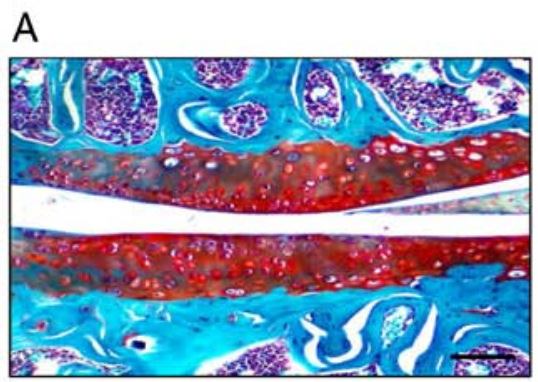

Sham/vehicle

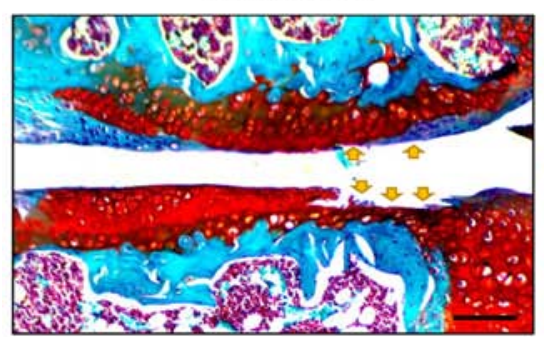

OA/vehicle

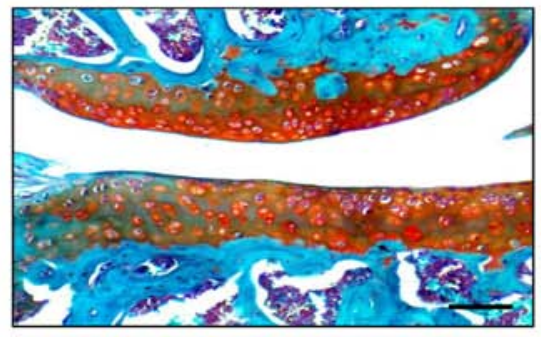

Sham/ER

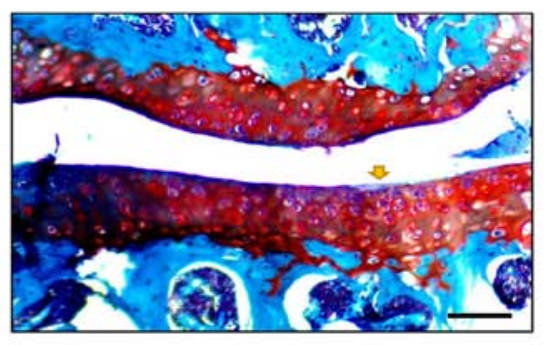

OA/ER
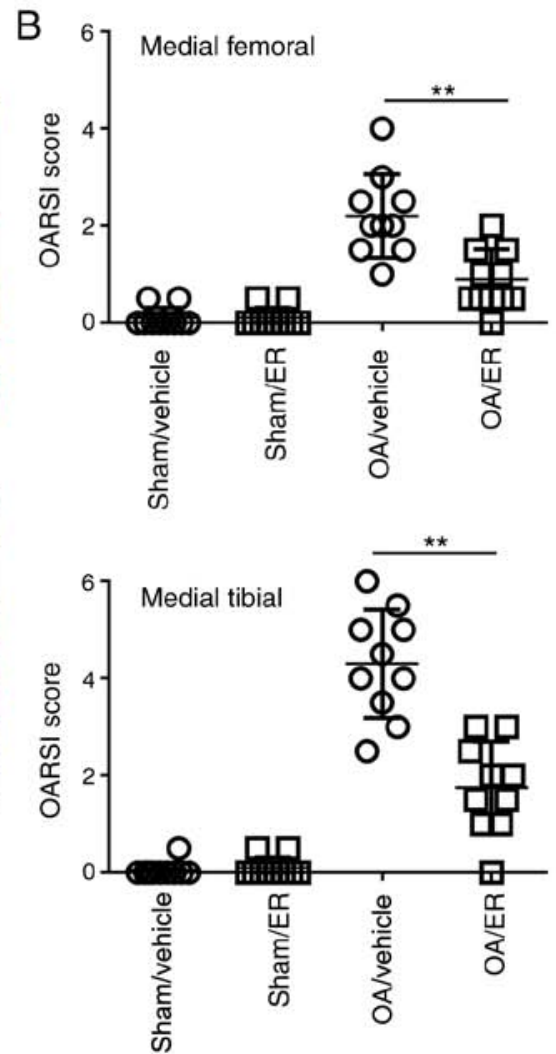

Figure 3. ER protects against OA. The right knees of mice underwent destabilization of the medial meniscus surgery and the left knees underwent sham surgery. Subsequently, mice were administered with ER or vehicle for 2 weeks after surgery. $n=10 /$ group. (A) Representative sections of knee joints from experimental mice stained with Safranin O/fast green. Yellow arrows indicate destruction-affected cartilage areas. (B) Histological scoring of OA. The scores were for femoral and tibial samples on the $\mathrm{y}$-axes of representative graphs. ${ }^{* *} \mathrm{P}<0.01$ as indicated. ER, ergosterol; OA, osteoarthritis; vehicle, saline. 

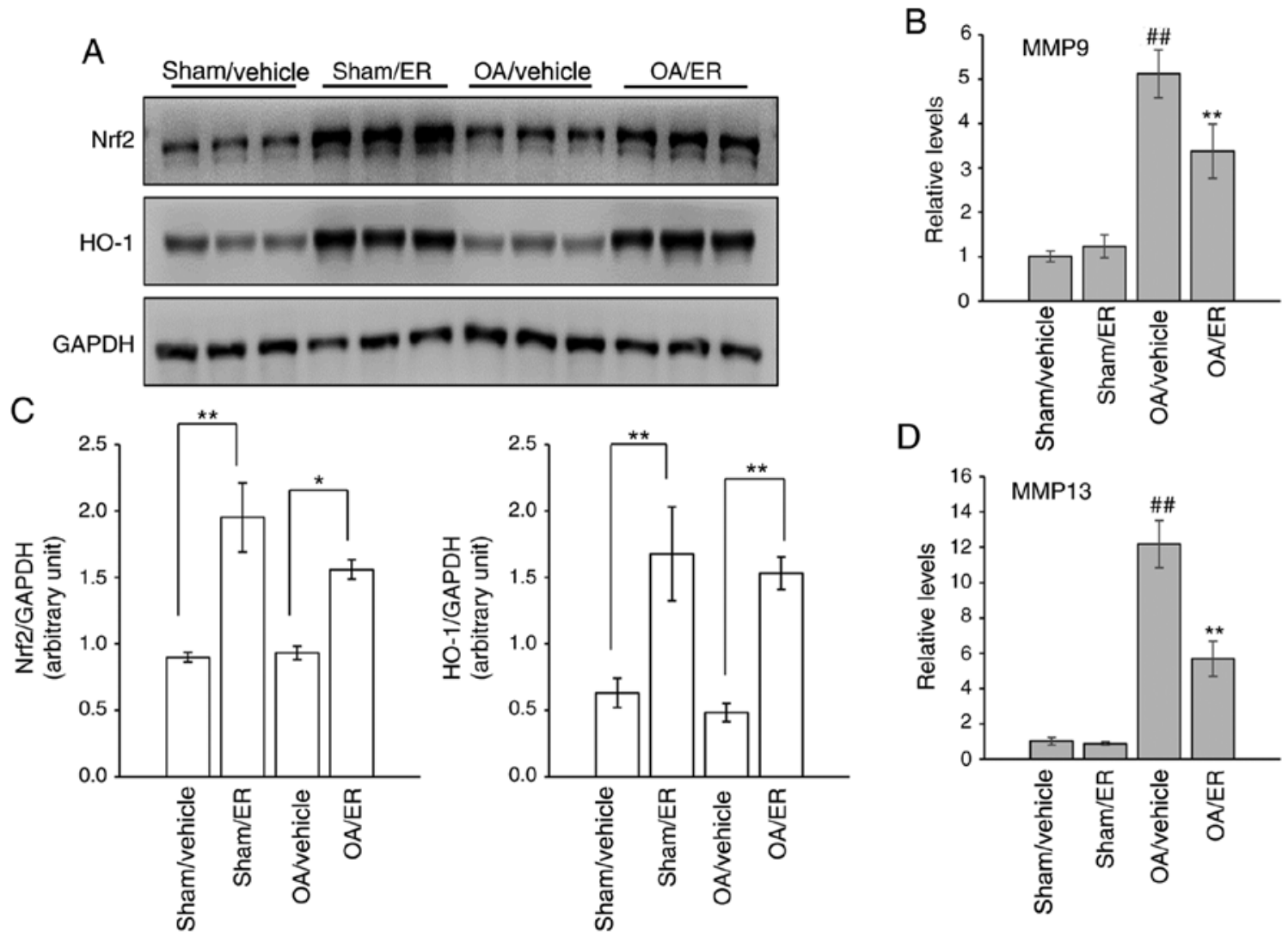

Figure 4. Effects of ER on the protein expression levels of Nrf2, HO-1, and the mRNA expression levels of MMP-9 and MMP-13 in experimental OA. (A) Western blot analysis of Nrf2 and HO-1 expression in the cartilage from murine knee joints. (B) mRNA expression of MMP-9. ${ }^{\# \#} \mathrm{P}<0.01 \mathrm{vs.} \mathrm{the} \mathrm{sham/vehicle}$ group. ${ }^{* *} \mathrm{P}<0.05$ vs. OA/vehicle group. (C) Quantitative analysis of Nrf2 and $\mathrm{HO}-1$ protein levels. ${ }^{*} \mathrm{P}<0.05$ and ${ }^{* *} \mathrm{P}<0.01$ as indicated. (D) mRNA expression of MMP-13. ${ }^{\# /} \mathrm{P}<0.01$ vs. the sham/vehicle group. ${ }^{* *} \mathrm{P}<0.01$ vs. OA/vehicle group. ER, ergosterol; Nrf2, nuclear factor erythroid 2-related factor 2; HO-1, heme oxygenase 1; DMM, destabilization of the medial meniscus; OA, osteoarthritis.

ligament. ER (25 mg/kg/day) or vehicle was administered for 2 weeks post-operation. Mice were sacrificed at 8 weeks post-DMM surgery. The knee joint tissues were collected for Safranin O/Fast Green staining. Histological sections were assessed using OARSI scores in a blinded manner. The data showed that the ER-treated group significantly improved femur and tibia proteoglycan loss or cartilage damage compared with the vehicle-treated group at 8 weeks post-DMM surgery. Knee cartilage harvested from mice administrated with ER or vehicle that underwent sham surgery showed no damage, indicating the ER administration had no effect on an undamaged knee. These results suggested that that oral administration of ER in mice effectively delayed the progression of OA (Fig. 3A and B).

ER promotes expression of $\mathrm{Nrf2/HO}-1$ and suppresses gene expression of MMPs in experimental OA. The expression levels of Nrf2 and HO-1 in the cartilage of knee joints were measured by western blot analysis to determine whether activation of the Nrf2 signaling pathway in response to ER was similar to that observed in vitro. The results showed that protein expression levels of Nrf2 and HO-1 were increased in the cartilage of mice administrated orally with ER compared with the respective vehicle-treated groups (Fig. 4A and C). The effect of ER on the expression of MMP-9 and MMP-13 in articular cartilage was further assessed. Gene transcript analysis results showed that the mRNA expression levels of
MMP-9 and MMP-13 were increased in the knee cartilage from the OA/vehicle group compared with the sham/vehicle group, and this upregulation was suppressed by ER (Fig. 4B and D), similar to the aforementioned in vitro assay results.

\section{Discussion}

Excessive oxidative stress is associated with OA and triggers chondrocyte senescence and apoptosis, extracellular matrix (ECM) degradation, dysfunction of the subchondral bone and synovial inflammation during OA (27). The transcription factor Nrf2 regulates the expression of a set of genes that counteract oxidative stress. Under physiological conditions, Nrf2 is tethered in the cytoplasm by its inhibitor, Kelch-ECH associated protein 1 (KEAP1), which controls its proteasomal degradation. During increased oxidative or electrophilic stress, Nrf2 is released from KEAP1 and translocates to the nucleus, binding to AREs located in the promoter regions to activate its target genes, including HO-1, which is an important defense against reactive oxygen species-mediated damage in various tissue injuries (28). A previous study revealed that $\mathrm{HO}-1$ expression markedly decreased in the articular cartilage of wild-type mice with age, and that maintenance of HO-1 expression had the potential to protect against OA development (29). The present study confirmed that ER enhanced the nuclear translocation of Nrf2, promoted HO-1 promoter transactivation and upregulated the expression of genes downstream of Nrf2, 
indicating that ER activated the Nrf2 signaling pathway, which contributed to preventing cartilage degeneration.

Common medication for OA aims to relieve patient's joint pain of to improve quality of life $(30,31)$. Trichostatin A, a histone deacetylase inhibitor, was used to activate Nrf2 signaling pathways, subsequently leading to a significantly decreased severity of cartilage damage in DMM surgery-induced OA mice in our previous study (10). While disease-modifying OA drugs (DMOAD) require long-term use, the search for potential drugs targeting Nrf2 activation continues, considering the side effects caused by trichostatin A (32). In the present study, ergosterol significantly activated the Nrf2 pathway in primary chondrocytes. The present study further demonstrated that oral administration of ER in mice significantly increased the expression of Nrf2 and $\mathrm{HO}-1$ in murine knee cartilage, exerting protective effects on the cartilage during OA. Additionally, the oral administration of ER may make it more suitable for clinical use as a potential DMOAD in comparison with injectable therapeutic agents.

When measuring the expression of ECM-degrading proteases, the present study found that ER significantly inhibited the expression levels of MMP9 and MMP13 in chondrocytes and cartilage, which were enhanced during OA and play an important role in articular cartilage damage. Previous studies reported that upregulation of $\mathrm{Nrf} 2$ downstream proteins such as HO-1 can reduce the expression of MMPs and inhibit the production of proinflammatory cytokines (29,33-35). Hence, the inhibition of MMP9 and MMP13 may be partially attributed to ER-induced Nrf2 signaling activation.

Several limitations of the present study should be noted. First, the mice used in the experiments were relatively young ( $\sim 10$ weeks old) with immature skeletons. Therefore, the possibility that other late developmental events might affect the effectiveness of ER in the OA model could not be excluded. Second, since ER was only administered at the same time as OA onset in the experiment, it could not be determined whether ER was protective in pre-arthritic knees. A middle-stage OA model should be used in future investigations to fully elucidate the preventive effect of ER. Although to the best of our knowledge there are no direct links between ER and Nrf2 expression in the existing literature, with further research into the physiological effect of ER, more cross-pathways may be found to help understand the potential regulation of $\mathrm{Nrf} 2$ protein expression by ER. The present study made an assumption that ER may alter the expression of Nrf2 gene through epigenetics, such as microRNA (miRNA/miR). Previous studies have shown that miR-144, miR-28, miR-93 and other miRNAs can regulate Nrf2 gene expression (36-38), while miR-125a, miR-378 and other miRNAs have been shown to be regulated by ER (39-41). A possibility that ER mediated the expression of Nrf2 through certain miRNA cannot be ruled out in the present study.

In conclusion, the present study found that ER served a regulatory role in anti-oxidative damage and reduction of catabolism in cartilage tissues, suggesting that ER could be considered a promising effective option for the treatment of OA.

\section{Acknowledgements}

Not applicable.

\section{Funding}

The present study was supported by grants from the Natural Science Foundation of the Jiangsu Higher Education Institutions of China (grant no. 18KJB320009), Scientific Research Project of Hunan Education Department (grant no. 13C836) and Technological Innovation Guidance Plan of Hunan Province (grant no. 2017SK50214).

\section{Availability of data and materials}

The datasets used and/or analyzed during the current study are available from the corresponding author on reasonable request.

\section{Authors' contributions}

DC, XW and JQ conceived and designed the study. DC, HY, JL, SC and LJ performed the experiments. DC and HY wrote the paper. JQ and XW reviewed and edited the manuscript. All authors read and approved the final manuscript.

\section{Ethics approval and consent to participate}

All experiments performed with the use of animals were approved by the Animal Ethical and Welfare Committee of Nanjing Medical University Institutional Animal Care and Use Committee (approval no. IACUC 1903044).

\section{Patient consent for publication}

Not applicable.

\section{Competing interests}

The authors declare that they have no competing interests.

\section{References}

1. Li T, Ma J, Zhao T, Gao F and Sun W: Application and efficacy of extracorporeal shockwave treatment for knee osteoarthritis: A systematic review and meta-analysis. Exp Ther Med 18: 2843-2850, 2019.

2. Fragkiadaki P, Nikitovic D, Kalliantasi K, Sarandi E, Thanasoula M, Stivaktakis PD, Nepka C, Spandidos DA, Tosounidis $\mathrm{T}$ and Tsatsakis A: Telomere length and telomerase activity in osteoporosis and osteoarthritis. Exp Ther Med 19: 1626-1632, 2020

3. Joly DA, Ludwig T, Mahdavi S, Khong H, Piroozfar SG and Sharma R: Does age influence patient-reported outcomes in unilateral primary total hip and knee arthroplasty? J Arthroplasty 35: 1800-1805, 2020.

4. Park C, Hong SH, Shin SS, Lee DS, Han MH, Cha HJ, Kim S, Kim HS, Kim GY, Park EK, et al: Activation of the Nrf2/HO-1 signaling pathway contributes to the protective effects of sargassum serratifolium extract against oxidative stress-induced DNA damage and apoptosis in SW1353 human chondrocytes. Int J Environ Res Public Health 15: 1173, 2018.

5. Vaamonde-Garcia C, Courties A, Pigenet A, Laiguillon MC, Sautet A, Houard X, Kerdine-Römer S, Meijide R, Berenbaum F and Sellam J: The nuclear factor-erythroid 2-related factor/heme oxygenase- 1 axis is critical for the inflammatory features of type 2 diabetes-associated osteoarthritis. J Biol Chem 292: 14505-14515, 2017.

6. Alcaraz MJ and Ferrandiz ML: Relevance of Nrf2 and heme oxygenase-1 in articular diseases. Free Radic Biol Med 157: 83-93, 2020. 
7. Li X, Lin J, Ding X, Xuan J, Hu Z, Wu D, Zhu X, Feng Z, Ni W and $\mathrm{Wu} \mathrm{A}$ : The protective effect of sinapic acid in osteoarthritis: In vitro and in vivo studies. J Cell Mol Med 23: 1940-1950, 2019.

8. Qiao YQ, Jiang PF and Gao YZ: Lutein prevents osteoarthritis through Nrf2 activation and downregulation of inflammation. Arch Med Sci 14: 617-624, 2018.

9. Robledinos-Antón N, Fernández-Ginés R, Manda G and Cuadrado A: Activators and inhibitors of NRF2: A review of their potential for clinical development. Oxid Med Cell Longev 2019: 9372182, 2019.

10. Cai D, Yin S, Yang J, Jiang Q and Cao W: Histone deacetylase inhibition activates Nrf2 and protects against osteoarthritis. Arthritis Res Ther 17: 269, 2015.

11. Landolfo S,Zara G,Zara S, Budroni M, Ciani M and Mannazzu I: Oleic acid and ergosterol supplementation mitigates oxidative stress in wine strains of Saccharomyces cerevisiae. Int J Food Microbiol 141: 229-235, 2010.

12. Yasukawa K, Aoki T, Takido M, Ikekawa T, Saito H and Matsuzawa T: Inhibitory effects of ergosterol isolated from the edible mushroom Hypsizigus marmoreus on TPA-induced inflammatory ear oedema and tumour promotion in mice. Phytother Res 8: 10-13, 1994.

13. Xu J, Lin C, Wang T, Zhang P, Liu Z and Lu C: Ergosterol attenuates LPS-induced myocardial injury by modulating oxidative stress and apoptosis in rats. Cell Physiol Biochem 48: 583-592, 2018.

14. Cai D, Feng W, Liu J, Jiang L, Chen S, Yuan T, Yu C, Xie H, Geng D and Qin J: 7,8-Dihydroxyflavone activates Nrf2/HO-1 signaling pathways and protects against osteoarthritis. Exp Ther Med 18: 1677-1684, 2019

15. Shakibaei M, John T, Seifarth C and Mobasheri A: Resveratrol inhibits IL-1 beta-induced stimulation of caspase- 3 and cleavage of PARP in human articular chondrocytes in vitro. Ann N Y Acad Sci 1095: 554-563, 2007.

16. Zhou PH, Liu SQ and Peng H: The effect of hyaluronic acid on IL-1beta-induced chondrocyte apoptosis in a rat model of osteoarthritis. J Orthop Res 26: 1643-1648, 2008.

17. Cai D, Huff TW, Liu J, Yuan T, Wei Z and Qin J: Alleviation of cartilage destruction by sinapic acid in experimental osteoarthritis. Biomed Res Int 2019: 5689613, 2019.

18. Lorenz H, Wenz W, Ivancic M, Steck E and Richter W: Early and stable upregulation of collagen type II, collagen type I and YKL40 expression levels in cartilage during early experimental osteoarthritis occurs independent of joint location and histological grading. Arthritis Res Ther 7: R156-R165, 2005

19. Livak KJ and Schmittgen TD: Analysis of relative gene expression data using real-time quantitative PCR and the 2(-Delta Delta C(T)) method. Methods 25: 402-408, 2001.

20. Sherf BA, Navarro SL, Hannah RR and Wood KV: Dual-luciferase reporter assay: an advanced co-reporter technology integrating firefly and Renilla luciferase assays. Promega Notes 57: 2-9, 1996

21. Glasson SS, Blanchet TJ and Morris EA: The surgical destabilization of the medial meniscus (DMM) model of osteoarthritis in the 129/SvEv mouse. Osteoarthritis Cartilage 15: 1061-1069, 2007.

22. Glasson SS, Chambers MG, Van Den Berg WB and Little CB: The OARSI histopathology initiative-recommendations for histological assessments of osteoarthritis in the mouse. Osteoarthritis Cartilage 18 (Suppl 3): S17-S23, 2010.

23. Zeng GQ, Chen AB, Li W, Song JH and Gao CY: High MMP-1, MMP-2, and MMP-9 protein levels in osteoarthritis. Genet Mol Res 14: 14811-14822, 2015.

24. Zhou X, Cao H, Yuan Y and Wu W: Biochemical signals mediate the crosstalk between cartilage and bone in osteoarthritis. Biomed Res Int 2020: 5720360, 2020.

25. Li H, Wang D, Yuan Y and Min J: New insights on the MMP-13 regulatory network in the pathogenesis of early osteoarthritis. Arthritis Res Ther 19: 248, 2017.
26. Chen J, Wang C, Huang K, Chen S and Ma Y: Acacetin suppresses IL-1 $\beta$-induced expression of matrix metalloproteinases in chondrocytes and protects against osteoarthritis in a mouse model by inhibiting NF- $\mathrm{KB}$ signaling pathways. Biomed Res Int 2020: 2328401, 2020

27. Lepetsos $P$ and Papavassiliou AG: ROS/oxidative stress signaling in osteoarthritis. Biochim Biophys Acta 1862: 576-591, 2016.

28. Ahmed SM, Luo L, Namani A, Wang XJ and Tang X: Nrf2 signaling pathway: Pivotal roles in inflammation. Biochim Biophys Acta Mol Basis Dis 1863: 585-597, 2017.

29. Takada T, Miyaki S, Ishitobi H, Hirai Y, Nakasa T, Igarashi K, Lotz MK and Ochi M: Bach1 deficiency reduces severity of osteoarthritis through upregulation of heme oxygenase-1. Arthritis Res Ther 17: 285, 2015.

30. Wang L, Chen H, Lu H, Wang Y, Liu C, Dong X, Chen J, Liu N, Yu F, Wan Q and Shang S: The effect of transtheoretical model-lead intervention for knee osteoarthritis in older adults: A cluster randomized trial. Arthritis Res Ther 22: 134, 2020.

31. Zhong HM, Zhao GF, Lin T, Zhang XX, Li XY, Lin JF, Zhao SQ and Pan ZJ: Intra-articular steroid injection for patients with hip osteoarthritis: A systematic review and meta-analysis. Biomed Res Int 2020: 6320154, 2020.

32. Im GI and Choi YJ: Epigenetics in osteoarthritis and its implication for future therapeutics. Expert Opin Biol Ther 13: 713-721, 2013.

33. Park SY, Jin ML, Kim YH, Lee SJ and Park G: Sanguinarine inhibits invasiveness and the MMP-9 and COX-2 expression in TPA-induced breast cancer cells by inducing HO-1 expression. Oncol Rep 31: 497-504, 2014

34. Rousset F, Nguyen MV, Grange L, Morel F and Lardy B: Heme oxygenase-1 regulates matrix metalloproteinase MMP-1 secretion and chondrocyte cell death via Nox4 NADPH oxidase activity in chondrocytes. PLoS One 8: e66478, 2013.

35. Lee IT, Luo SF, Lee CW, Wang SW, Lin CC, Chang CC, Chen YL, Chau LY and Yang CM: Overexpression of HO-1 protects against TNF-alpha-mediated airway inflammation by down-regulation of TNFR1-dependent oxidative stress. Am J Pathol 175: 519-532, 2009.

36. Li B, Zhu X, Ward CM, Starlard-Davenport A, Takezaki M, Berry A, Ward A, Wilder C, Neunert C, Kutlar A and Pace BS: MIR-144-mediated NRF2 gene silencing inhibits fetal hemoglobin expression in sickle cell disease. Exp Hematol 70: 85-96. e5, 2019.

37. Yang M, Yao Y, Eades G, Zhang Y and Zhou Q: MiR-28 regulates Nrf2 expression through a Keap1-independent mechanism. Breast Cancer Res Treat 129: 983-991, 2011.

38. Singh B, Ronghe AM, Chatterjee A, Bhat NK and Bhat HK: MicroRNA-93 regulates NRF2 expression and is associated with breast carcinogenesis. Carcinogenesis 34: 1165-1172, 2013.

39. Wang L, Yang Y and Hong B: Advances in the role of microRNAs in lipid metabolism-related anti-atherosclerotic drug discovery. Expert Opin Drug Discov 8: 977-990, 2013.

40. Croston TL,Lemons AR, Beezhold DH and Green BJ: MicroRNA regulation of host immune responses following fungal exposure. Front Immunol 9: 170, 2018.

41. Wu QP, Xie YZ, Deng Z, Li XM, Yang W, Jiao CW, Fang L, Li SZ, Pan HH, Yee AJ, et al: Ergosterol peroxide isolated from Ganoderma lucidum abolishes microRNA miR-378-mediated tumor cells on chemoresistance. PLoS One 7: e44579, 2012.

This work is licensed under a Creative Commons Attribution-NonCommercial-NoDerivatives 4.0 International (CC BY-NC-ND 4.0) License. 